\title{
SOLITARY FIBROUS TUMOUR OF THE ORBIT: A CASE REPORT
}

\author{
Vaibhav Mane, S. R. Agashe, R. T. Joshi, P. P. Patil
}

\author{
1. Assistant Professor. Department of Pathology, Bharati Vidyapeeth University's Medical College \& \\ Hospital,Sangli. \\ 2. Professor. Department of Pathology, Bharati Vidyapeeth University's Medical College \& Hospital,Sangli. \\ 3. Professor. Department of Pathology, Bharati Vidyapeeth University's Medical College \& Hospital,Sangli. \\ 4. Professor \& HOD. Department of Pathology, Bharati Vidyapeeth University's Medical College \& \\ Hospital,Sangli.
}

\section{CORRESPONDING AUTHOR:}

Dr. Vaibhav Mane,

Vaishnavi Pathology Laboratory,

Shop No.2 Kore House, Vishrambag,

Sangli. Maharashtra-416415.

E-mail: vaishnavilab@rediffmail.com

ABSTRACT: Solitary fibrous tumour is a rare spindle cell neoplasm. It was first described by Klemper and Rabin in 1931.Initially it was assumed that these tumours .The tumour originates from mesenchyme. The diagnosis is confirmed by immunohistochemical confirmation for CD 34 . These tumours usually have good prognosis. These tumours are treated with surgical excision with close followup Local recurrences were seen after incomplete surgical excision

KEY WORDS: Hemangiopericytoma, Orbit, Proptosis, Solitary fibrous tumour, CD -34

INTRODUCTION: Solitary fibrous tumours (SFTs) are uncommon spindle cell tumours of mesenchyme. Commonly these tumours are seen in pleura, lungs, peritoneum, pericardium, kidneys, liver and rarely in head and neck region. $(1,3)$ Orbital solitary fibrous tumours are less common benign, slow growing presents with painless proptosis. $(1,3,4,6)$ On immunohistochemistry these are positive for CD34 and vimentin. (1, 2, 3, 4, 6) Usually these tumours are treated with complete excision with no recurrence. $(3,4)$

CASE REPORT: A 42-year-old male patient was admitted with complains of left eye proptosis since last six months. On examination left eye axial proptosis. No deficit of visual activity . Elevation of left eye restricted.USG, CT scan and Left eye B scan demonstrated a well defined soft tissue mass in intraconal compartment in left eye retrobulbar area. The patient underwent orbitotomy with complete removal of the mass. The postoperative period was uneventful with complete resolution of proptosis.

Routine laboratory test were within normal ranges. Gross examination showed a tan colored mass. Cut section shows areas of haemorrhage microscopically the tumour showed alternating areas of hypo and hypercellularity. It shows fibrocollagenous tissue and spindle shaped cells arranged haphazardly i.e. pattern less pattern. Few vessels were also noted. Histological diagnosis of solitary fibrous tumour of orbit was given. Immunohistochemistry showed the cells were positive CD 34 .

DISCUSSION: The first orbital SFTs were described by Dorfman et al and Westra et al, in 1994 . It is a tumor of mesenchymal origin that usually arises in the pleura. (1) SFT in other extrapleural sites, including the lung, mediastinum, peritoneum, upper respiratory tract, thyroid and liver has been increasingly reported in recent years. (1) 
Orbital SFT most commonly presents in middle-aged patients with gradual unilateral progressive proptosis and mass effect. Predominantly located in the superior part of the orbit, it is usually unassociated with features of pain or entrapment like nerve palsies. (3)

The diagnosis of SFT rests on the histological appearance and the immunohistochemical confirmation. One classic feature is the presence of spindle cells that grow in a haphazard fashion in a variably cellular stroma, known as the "patternless pattern". The stroma is often heavily collagenized, with bands of collagen interspersed between the tumor cells. $(1,2,3)$

The cells are often densely arranged in a fascicular and storiform pattern with numerous vascular channels that may occasionally form branching or stag horn-like channels. (3) The histopathologic differential diagnosis is giant cell angiofibroma, fibrous histiocytoma, hemangiopericytoma, peripheral nerve sheath tumour, fibromyxoid sarcoma. (1)

SFT are reactive for mesenchymal markers like vimentin. (1) Immunoreactivity with marker CD 34 is present in $75-100 \%$ of cases. (1)

The CT and MRI characteristics of SFTs of the orbit illustrate a well-defined, enhanced mass lesion. (2) CT scan typically reveals a mild to moderately enhancing, well-circumscribed mass with or without local bony remodeling. (3)

Intense CD34 immunohistochemical staining helps to differentiate SFTs from hemangiopericytoma, which shows weak and patchy CD34 staining, and also from fibrous histiocytoma, which shows CD34 negative. Schwannoma is also CD34-positive, but it is very focal, and it will be strongly positive for neural markers such as S-100 protein. (2)SFTs are negative to desmin, cytokeratin, factor VIII-related antigen, S-100, SMA, and muscle-specific actin. (3)

Orbital SFTs are largely benign tumors with few instances of recurrence. Most reported cases behave in a nonaggressive fashion, similar to SFT in other sites in the head and neck region. ${ }^{(3)}$ The clinical behavior is variable. Most SFTs are benign, but local invasion or recurrence of the lesion has been demonstrated; in a few cases. (2)

Current management of solitary fibrous tumors of the orbit is surgical resection with periodic follow-up to monitor for recurrence. $(1,2,3,4,5)$

CONCLUSION: Solitary fibrous tumours of the orbit are uncommon benign tumours, usually presenting with unilateral painless proptosis in middle age persons. The CT and MRI characteristics of SFTs of the orbit illustrate a well-defined, enhanced mass lesion. Histological diagnosis with IHC is must. And complete surgical excision with adequate margins with close followup is the treatment.

\section{REFERENCES:}

1. Mukherjee B, Biswas J. Solitary fibrous tumor of the orbit. Indian J Pathol Microbiol 2008;51:453-5. http://www.ijpmonline.org/text.asp?2008/51/3/453/42530

2. Shu-Shong Hsu,Ping-Hong Lai,Jyh-Seng Wang,Chi-Man Yip, J Chin Med Assoc 2004;67:483-486

3. Mohammad Javed Ali, Santosh G. Honavar, Milind N. Naik, and Geeta K. Vemuganti. Orbital solitary fibrous tumor: A clinicopathologic correlation and review of literature. Oman J Ophthalmol. 2011 Sep-Dec; 4(3): 147-149.

4. Peter A.D. Rubin, Gabriel Garza, Mark P. Hatton; 44 year old man with incidental orbital mass, Digital Journal of Ophthalmology 2002, Volume 8, Number 2 
5. Hayashi, Satoru M.D.; Kurihara, Hideyuki M.D.; Hirato, Junko M.D.; Sasaki, Tomio M.D. Solitary Fibrous Tumor of the Orbit with Extraorbital Extension: Case Report . Neurosurgery: November 2001 - Volume 49 - Issue 5 - pp 1241-1245

6. I.Arturi, D.Bollero, A.Pucci, R.Ferrero and M.Hamedani. Solitary fibrous tumour of the orbit :clinical and histological evidence. European Journal of Plastic Surgery, Volume 26, Number 8, February 2004 , pp. 419-421(3)

7. Giuffrè I, Faiola A, Bonanno E, Liccardo G. Solitary fibrous tumor of the orbit. Case report and review of the literature. Surg Neurol. 2001 Oct; 56(4):242-6.

8. Rob Melendez, Andrew Doan. Solitary fibrous tumour: 58 year old man with painless mass in orbit. Pearls in Opthalmology Volume 3, Issue 2 - July 2008 
Figure 1: Microphotograph showing haphazardly arranged spindle shaped cells and few blood vessels ( $\mathrm{H} \&$ E stain, Low Power ).

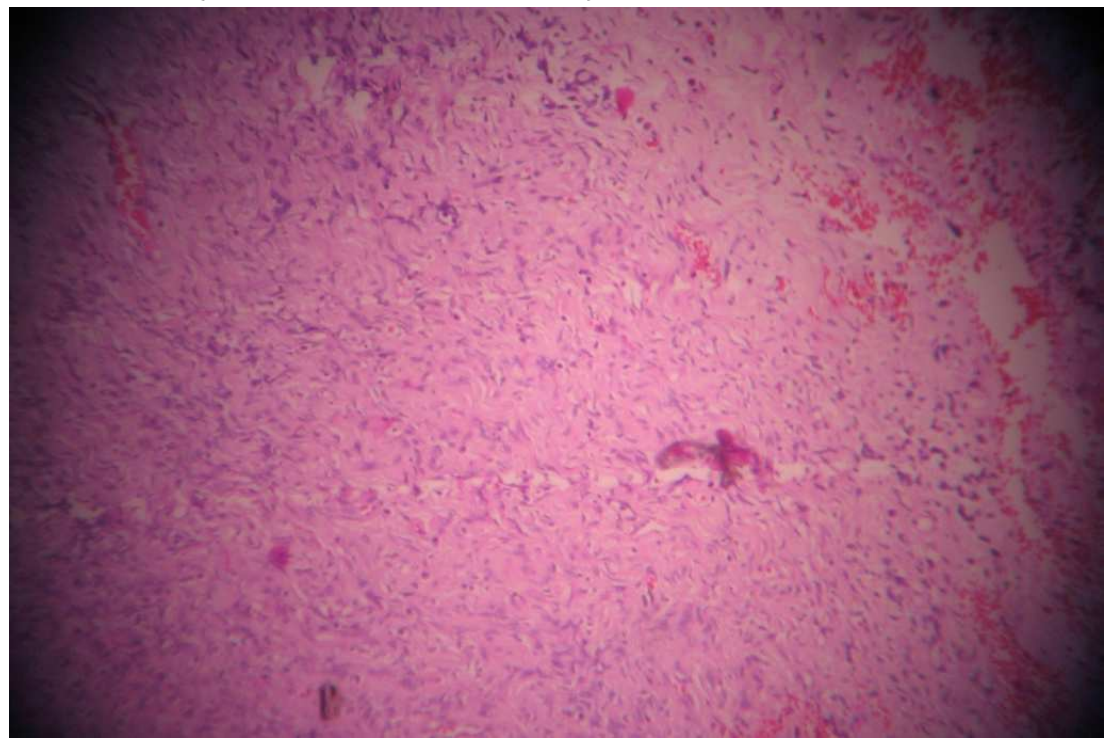

Figure 2: Microphotograph showing haphazardly arranged spindle shaped cells

(H \& E stain, High Power ).

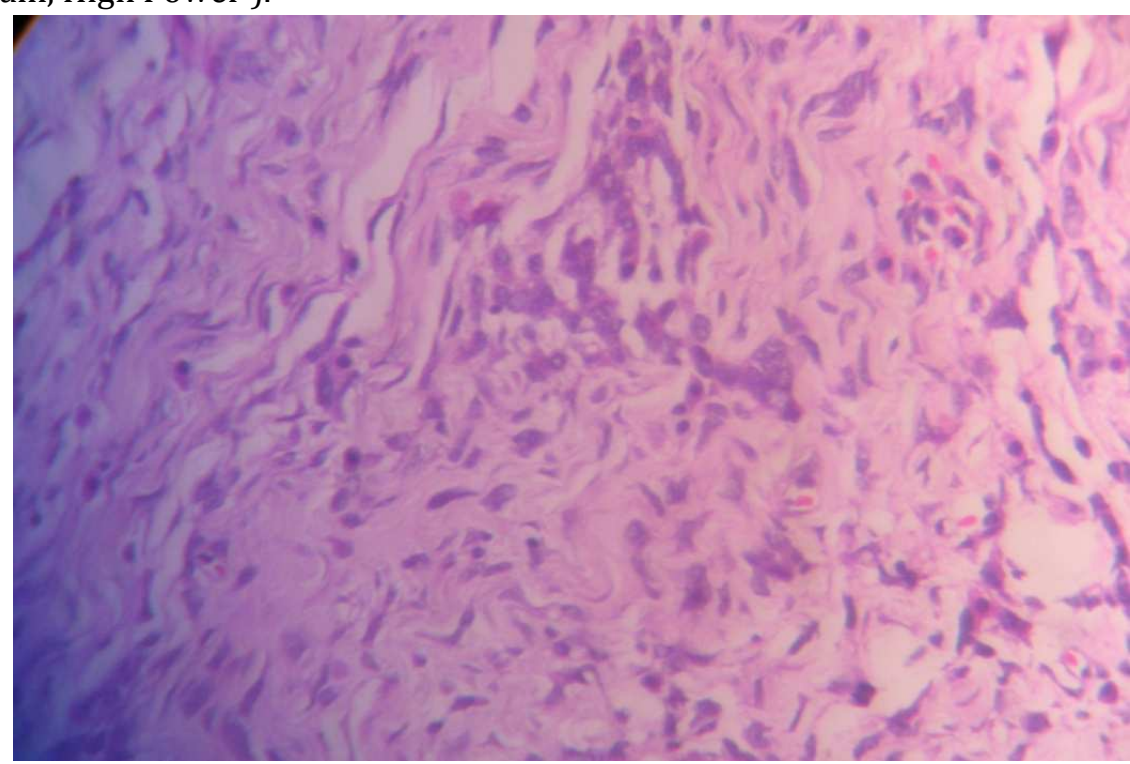

